Research Article

\title{
Processing and Properties of MDF Fibre-Reinforced Biopolyesters with Chain Extender Additives
}

\author{
Armin Thumm, ${ }^{1}$ Damien Even, ${ }^{2}$ Pierre-Yves Gini, ${ }^{3}$ and Mathias Sorieul $\mathbb{D}^{1}$ \\ ${ }^{1}$ Scion, Private Bag 3020, Rotorua 3046, New Zealand \\ ${ }^{2}$ Cabot Performance Materials, 78 Rue Prévochamps, Pepinster B-4860, Belgium \\ ${ }^{3}$ Safran Nacelles, Route du Pont 8, 76700 Gonfreville l'Orcher, France \\ Correspondence should be addressed to Mathias Sorieul; mathias.sorieul@scionresearch.com
}

Received 23 May 2018; Revised 24 September 2018; Accepted 5 November 2018; Published 16 December 2018

Guest Editor: Simona Zuppolini

Copyright ( 2018 Armin Thumm et al. This is an open access article distributed under the Creative Commons Attribution License, which permits unrestricted use, distribution, and reproduction in any medium, provided the original work is properly cited.

Biopolyesters are a way to improve natural fibre composite sustainability. This study explores, for the first time, the potential of using medium density fibreboard (MDF) fibres to reinforce four biobased and biodegradable polyester matrices to create a fully "green composite." Added at $30 \mathrm{wt} \%$, MDF fibres did not improve the strength of the injection moulded NFCs and this deficiency was investigated by measuring fibre length, viscosity, and molecular weight of the matrices. Compared to other lignocellulosic fibres, the use of MDF fibres led to a molecular weight reduction of biopolyesters during processing. This effect was particularly striking for PLA. The addition of a chain extender enhanced the molecular weight of PLA and improved its processability. The tensile strength increase was correlated to a reduction of fibre pull-out, enabling the MDF fibre to fulfil its expected reinforcement role within the biopolyester composite.

\section{Introduction}

Reinforced plastic composites are a class of materials in which fillers of high modulus are added to a polymer matrix to increase its modulus. The market of composites using natural fillers as reinforcement is expanding. In the emerging field of plastic composites reinforced with biobased fillers, plant lignocellulosic materials are the most widely used. The main advantages of lignocellulosic fillers over traditional inorganic fillers are their low cost, lightweight, renewability, abundant availability, and biodegradability [1]. Composites containing lignocellulosic material can be divided into two categories depending on the morphology of the filler; wood plastic composites (WPC) contain wood flour, and natural fibre composites (NFC) are reinforced with bast/leaf or wood fibres. Wood fibres are one of the most promising lignocellulosic fibre types; they have the advantage of being nonseasonal and of consistent quality. Moreover, unlike wood flour, their high aspect ratio contributes to the strength and stiffness of the composite material when used as a filler
$[2,3]$. Fibre morphology and physicochemical properties have a strong effect on their reinforcing properties within the thermoplastic matrix [4]. Wood fibre characteristics mainly depend on the fibre origin and refining method. They are extracted from softwood or hardwood, and the main techniques used for the fibrillation/individualisation process are mechanical (e.g., thermomechanical pulps), chemical (e.g., kraft), or a combination of the two (e.g., chemi-thermomechanical pulp) [5].

MDF (medium density fibreboard) fibres are thermomechanical fibres where high temperatures around $170^{\circ} \mathrm{C}$ lead to cleavage of the fibres along the middle lamella, exposing a comparatively lignin-rich, nonpolar, fibre surface [6-8]. The typical MDF fibre from Pinus radiata is approximately $1.5 \mathrm{~mm}$ long and $34 \mu \mathrm{m}$ wide, with an aspect ratio of $44 / 1$ [9-11]. The large production plants devoted to MDF and the relatively low production cost of MDF fibre make it an attractive industrial choice as a filler for NFC.

The NFC market is projected to be US $\$ 5.8$ billion globally by 2021 [12]. Therefore, it is not surprising to observe 
the burgeoning of wood fibre products derived from pulping processes, appearing for the reinforcement of thermoplastic materials. These products include $\mathrm{NCell}^{\mathrm{TM}}$ (GreenCore Composites Inc., Canada); reSound NF (PolyOne, USA); CreaMix (CreaFill Fibers Corp., USA); Kareline ${ }^{\circledR}$ (Kareline Natural Composites, Finland); and ForMi (UPM, Finland).

The typical ranges of tensile strength, tensile modulus, and elongation at the break of commercial NFCs range between 12 and $47 \mathrm{MPa}, 0.8$ and $5.6 \mathrm{GPa}$, and 1.4 and $5.4 \%$, respectively (Table 1 ).

Due to their attractive mechanical properties and convenience for compounding, the petrochemical-based polyolefins, polyethylene (PE) and polypropylene (PP), are the primary resins currently used in NFCs [4]. However, the resulting products, a mix of synthetic and biobased materials, are only partially biodegradable and difficult to recycle.

Driven by the increasing environmental impact of traditional plastic and biobased and biodegradable plastics have attracted extensive interest $[12,14,15]$. The creation of a fully biodegradable NFC enables single use or medium term applications and easier end-of-life management. The use of biodegradable polymers in composites has been limited by their higher production costs, slow crystallization, poor stability, narrow processing windows, and incompatibility between hydrophobic polymer and hydrophilic wood fibre [4]. Nevertheless, biopolyesters are more polar in nature than PP or PE [4], which could make them more suitable for use with natural fibres. This feature leads to a better dispersion of the wood-based fillers in a biopolyester matrix compared to PP [13]. In contrast to the widespread use of specific compatibilisers, such as maleated PP (MA-PP) in polyolefins, their use with biopolyester (MA-PHA [16] and MA-PLA [17]) is still limited and noncommercial [18]. Unfortunately, no trends in the effectiveness of compatibilisers on PLA-based NFCs have been identified. However, as for PP [19], fibres with high cellulose content have good interfacial adhesion with PLA [20] without the need of a compatibiliser. The considerable variation in the mechanical properties seen in studies suggests that the grade of PLA, origin of wood, and processing methods are the main factors influencing the performance of the resultant composites [21]. The factors controlling fibre-resin interfacial properties are considered to be the strength of the interfacial adhesion [22] or the mechanical interlocking [23] between a wood and PLA. The results can be summarized as follows: depending on their origin and mode of preparation, wood fillers have mixed effects on tensile strength. An increase in wood filler content in PLA-based NFCs improves the stiffness and decreases the elongation at break [13].

While a large number of natural fibres, including wood fibre and nanocellulose, have been compounded with PLA [24], to our knowledge, no published study has investigated the use of MDF fibres to reinforce bioplastics.

The four main criteria taken into consideration for the selection of a NFC are physical characteristics, mechanical performance, environmental friendliness, and manufacturing cost [25]. The automotive sector is one of the main drivers for the industrial uptake of NFC. For this sector especially, the cost competitiveness and reliable sourcing
TABLE 1: Range of tensile properties of NFCs made with a biodegradable polymer compared to commercial PP and PEbased NFCs (with a wood filler content of 30-50 wt \%), adapted from $[4,13]$.

\begin{tabular}{lccc}
\hline $\begin{array}{l}\text { Composite } \\
\text { type }\end{array}$ & $\begin{array}{c}\text { Tensile } \\
\text { strength (MPa) }\end{array}$ & $\begin{array}{c}\text { Tensile Young's } \\
\text { modulus (GPa) }\end{array}$ & $\begin{array}{c}\text { Elongation at } \\
\text { break (\%) }\end{array}$ \\
\hline PLA-NFC & $37-77$ & $1.2-8.9$ & $1.0-3.1$ \\
PHA-NFC & $13-26$ & $0.4-5.9$ & $0.5-6.6$ \\
$\begin{array}{l}\text { PP-NFC } \\
\text { (commercial) }\end{array}$ & $18-47$ & $1.8-5.6$ & $1.6-5.4$ \\
$\begin{array}{l}\text { HDPE-NFC } \\
\text { (commercial) }\end{array}$ & $12-39$ & $0.8-4.7$ & $1.4-2.9$ \\
\hline
\end{tabular}

are crucial. Therefore, MDF fibres have the potential to be an ideal reinforcing agent for NFCs. This study investigates the possibility of creating an economically competitive, fully green NFC using MDF fibres as the reinforcing agent of biopolyester matrices. Peltola et al. [13] hypothesise that the lignin present in thermomechanically pulped (TMP) spruce fibres improves the fibre/PLA interface. Therefore, MDF fibres with a higher lignin content than TMP fibres and surface coated with lignin should be ideal to reinforce a PLA matrix.

\section{Materials and Methods}

\subsection{Materials}

2.1.1. Biopolyester and Additives. Four commercially available biodegradable/compostable polyesters with different mechanical and thermal properties were selected: polylactic acid (PLA), polybutylene succinate (PBS), polybutylene adipate-co-terephtalate (PBAT), and polybutylene succinateco-adipate (PBSA). The biopolyesters used in this study are described in Table 2 . Some properties of the selected polymers can be seen in Table 3.

The plastic additive BioAdimide ${ }^{\circledR} 500 X T$, typically used to increase the service life of biobased polyesters and to stabilize melt viscosity during processing, was obtained from Rhein Chemie (Rheinau GmbH, Germany). Joncryl ${ }^{\circledR}$ ADR4300 , a polymeric chain extender designed to reverse the degradation of condensation polymers, was supplied by BASF Corporation (Sturtevant, WI, USA). The dosage for both chain extenders was at the upper limit of the manufacturer's recommendations.

2.1.2. Lignocellulosic Material. The MDF fibres were produced from Pinus radiata wood chips at Scion's fibre processing plant (Rotorua, New Zealand). Their average length was $1.44 \mathrm{~mm}$, width $34 \mu \mathrm{m}$, and aspect ratio 42 , and the fines content was $5.7 \%$. Bleached kraft pulp was obtained from Carter Holt Harvey Pulp \& Paper Kinleith Mill (Oji Fibre Solutions, New Zealand). Flax fibre slivers, intended for use in textile fabrics, had an unknown source. Wood flour was obtained from Carter Holt Harvey Pulp \& Paper Kinleith Mill (New Zealand) and was sieved to be between 40 and 80 meshes. 
TABLE 2: Biopolyesters used in this study.

\begin{tabular}{lccccc}
\hline Name & Acronym & Reference & Grade & Company & Country \\
\hline Polylactic acid & PLA & 5051D (now 3052D) & Injection & NatureWorks & USA \\
Polybutylene succinate & PBS & Enpol G4560 J & Injection & Ire Chemical & Korea \\
Polybutylene adipate-co-terephthalate & PBAT & Enpol 8060 & Extrusion & Ire Chemical & Korea \\
Polybutylene succinate-co-adipate & PBSA & Bionolle 3020 & Injection & Showa Highpolymer & Japan \\
\hline
\end{tabular}

TABle 3: Properties as specified by the manufacturers. Glass transition temperature $\left(T_{\mathrm{g}}\right)$, melting point $\left(T_{\mathrm{m}}\right)$, Young's modulus $(E)$, stress at maximum load $\left(\mathrm{TS}_{\max }\right)$, and impact strength (IS). NI (not indicated by the manufacturer). Courtesy of the manufacturers.

\begin{tabular}{|c|c|c|c|c|c|c|}
\hline Polymer & $T_{\mathrm{g}}\left({ }^{\circ} \mathrm{C}\right)$ & $T_{\mathrm{m}}\left({ }^{\circ} \mathrm{C}\right)$ & $E(\mathrm{GPa})$ & $\mathrm{TS}_{\max }(\mathrm{MPa})$ & IS $\left(\mathrm{kJ} / \mathrm{m}^{2}\right)$ & $\operatorname{MFI}\left(\mathrm{g}^{*} 10 \mathrm{~min}^{-1}\right)$ \\
\hline PLA 3052 & $55 / 60$ & $145 / 160$ & 3.6 (flex) & 57 & 15.8 & 25 \\
\hline PBS & $-35 /-30$ & $112 / 117$ & $0.78 / 0.8$ (flex) & $40 / 45$ & $38 / 42 \cdot 10^{-3}$ & 25 \\
\hline PBAT & $-30 /-25$ & $127 / 132$ & $0.11 / 0.15$ (flex) & 44 & No break & 2 \\
\hline PBSA & -45 & 94 & 0.34 & 34 & NI & 25 \\
\hline
\end{tabular}

\subsection{Methods}

2.2.1. MDF Fibre Characterisation. Fibre size and fine content of the original MDF fibres were measured using an FQA-360 fibre analyser. The resolution of this instrument is $14 \mu \mathrm{m} /$ pixel (OpTest Equipment Inc., Canada).

2.2.2. Extrusion Compounding. The polymers and $\mathrm{MDF}$ fibres were dried overnight at $50^{\circ} \mathrm{C}$ and $105^{\circ} \mathrm{C}$, respectively, before extrusion compounding. Polymers were combined with fibres in a twin screw LABtech ${ }^{\mathrm{TM}}$ extruder type LTE 26-40 (26 mm corotating screws; $1 / d=40$ ) (LabTech Engineering Co. Ltd., Praksa, Muang, Samutprakarn, Thailand) with an atmospheric vent used to vent volatiles. The polymer was gravimetrically fed into the main feed. Simultaneously, the fibre was hand fed at the same port to obtain a $30 \mathrm{wt} \%$ fibre content. The fibre content was selected due to its wide market application [26]. The compounded materials were extruded at $180^{\circ} \mathrm{C}$ using a rotational screw speed of $150 \mathrm{rpm}$. The total feed rate was $2.5 \mathrm{kgh}^{-1}$ for the MDF fibres; all other materials' feed rate was $5 \mathrm{kgh}^{-1}$. The compound went through a two-strand die and was water cooled before being pelletized into $3 \mathrm{~mm}$ long wood plastic pellets.

2.2.3. Injection Moulding. The virgin biopolyester was extruded into plain or wood plastic strands and pelletised. The pellets were dried overnight in an oven at $60^{\circ} \mathrm{C}$ for the PLA composites and $80^{\circ} \mathrm{C}$ for the PBS, PBSA, and PBAT. The dried pellets were injection moulded on a BOY 35 machine (BOY Spritzgiessautomaten, Neustadt-Fernthal, Germany) into tensile and flexural specimens (sample shape according to ASTM D 638-03 type I). Injection moulding settings had to be adjusted according to the formulation in order to completely fill the mould cavities without flash and to produce satisfactory test specimens. The PLA, PBS, and PBAT composites were injection moulded at $180^{\circ} \mathrm{C}$, and the PBSA samples at $110^{\circ} \mathrm{C}$. A mould temperature of $30^{\circ} \mathrm{C}$ was chosen in order to obtain short cycle times. A cooling time of 20 seconds was used for all polymers except PBAT, where
10 seconds were used due to a longer packing time. Composite densities were measured to ensure that the filler loading was accurate and no voids were present in the composites.

2.2.4. Tensile Properties. Tensile specimens were tested using an Instron 5566 machine (Instron, USA). The tensile properties Young's modulus and maximum strength were obtained from stress-strain curves according to ASTM D638 [27] $(n=10)$.

2.2.5. Impact Strength. Notched Izod impact specimens were tested using a CEAST Resil impactor 6957 (CCSi, USA) according to ASTM 256 [28]. Ten specimens of each composite were tested to obtain the impact strength average value.

2.2.6. Molecular Weight Measurements. Gel permeation chromatography (GPC) was used to determine the molecular weight distribution of the plain and processed biopolyesters. GPC was carried out on a PL-GPC-50 system (Polymer Laboratories, UK, now Varian Inc.) with a Knauer K-301 refractive index detector using two PLgel Mixed-E $(300 \times 7.5 \mathrm{~mm}$, $3 \mu \mathrm{m})$ columns connected in series and protected by a guard column $(50 \times 7.5 \mathrm{~mm})$ of the same material. The system was run at $30^{\circ} \mathrm{C}$ with analytical grade chloroform as eluent. Flow rate was maintained at $1 \mathrm{~mL}^{*} \mathrm{~min}^{-1}$. The system was calibrated using polystyrene standards. Composite samples were stirred with chloroform for four hours at room temperature and filtered to remove the wood fibre (Watermann, ashless 40). The biopolyesters were then recovered with a Rotovapor ${ }^{\circledR}$. Two technical repeats were taken from each extract and prepared with a chloroform concentration of $10 \mathrm{mg} \mathrm{mL}^{-1}$. Molecular weights are expressed as the weight-average molecular weight (MW) which is defined as

$$
\mathrm{MW}=\frac{\sum_{i=1}^{N}\left(h_{i} M_{i}\right)}{\sum_{i=1}^{N} h_{i}},
$$



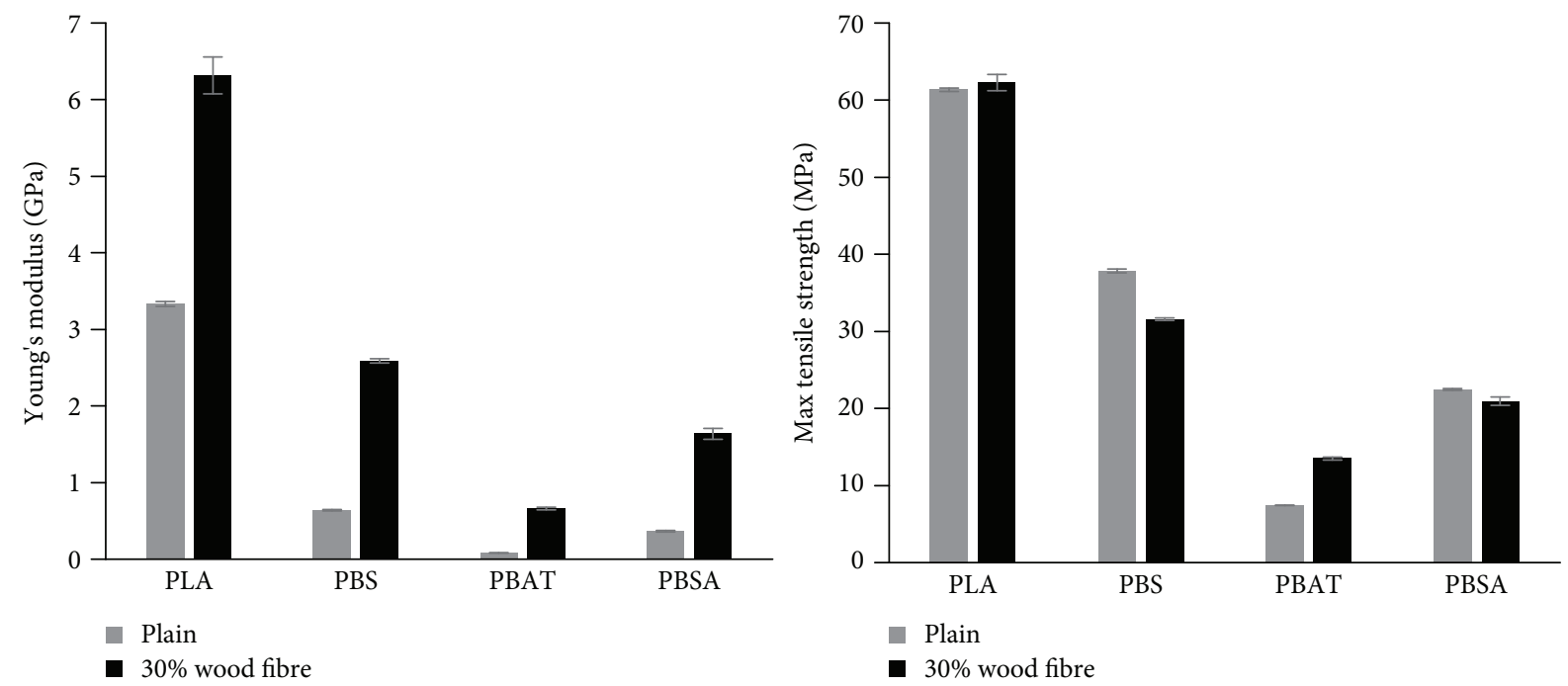

Figure 1: Tensile modulus and maximum tensile strength of injection moulded test samples with four biopolymers, plain or filled with 30 wt $\%$ MDF fibre (error bars $= \pm 95 \%$ confidence interval) $(n=10)$.

where $h_{i}$ is the height response of the concentration detector and $M_{i}$ is the molecular weight at $i$ th retention volume increment

2.2.7. Fibre Characterisation. The fibres were extracted from composites by chloroform extraction. One gram of sample was extracted using a Soxhlet apparatus with $200 \mathrm{~mL}$ of chloroform added. Samples were first boiled in the chloroform for two hours and then extracted in a Soxhlet for four hours under reflux.

The length of the extracted fibres was measured using a Fiberscan instrument (Andritz-Sprout-Bauer). The lengthweighted fibre length (LWL) average was used to compare samples. It puts a greater weight on longer fibres and is given by

$$
\mathrm{LWL}=\frac{\sum x^{2}}{\sum x},
$$

where $x$ is the individual fibre length.

The measurements, including fibre extraction, were performed in triplicate. A rayon standard of known length and distribution (Kajaani, \#2) was used to ensure instrument performance.

2.2.8. Rheology. The rheological properties of the materials were assessed in dynamic oscillation mode using a strain controlled rheometer AR2000 (TA instruments). The rheometer was equipped with $25 \mathrm{~mm}$ parallel plate geometry and an environmental test chamber to control the temperature. The compounded materials were dried in an oven at $103^{\circ} \mathrm{C}$ for four hours prior testing. To evaluate the thermal stability of the various compounds, the complex viscosity was monitored as a function of time. The time sweep measurements were conducted at $180^{\circ} \mathrm{C}$ using a strain of $2 \%$, frequency of $1 \mathrm{~Hz}$, and measurement gap of $1 \mathrm{~mm}$.
2.2.9. Microscopy. A fracture surface was prepared for each treatment, from notched impact testing samples. Samples were sputter coated with chromium using an Emitech K575X coater and imaged using a Jeol JSM 6700F field emission scanning electron microscope (SEM). Images were recorded at $5 \mathrm{kV}$ acceleration voltage and a probe current of $10 \mu \mathrm{A}$. The entire fracture surface was assessed, and then a representative area was imaged at 50x and 200x magnifications.

\section{Results and Discussion}

3.1. Mechanical Properties. The effect of MDF fibre on the tensile properties of four biopolyesters was tested. As expected, the stiffness of the four NFCs was increased by up to sixfold compared to the plain polyesters. However, the strength of the composites was not significantly changed for PLA and even decreased slightly for PBS and PBSA (Figure 1). The strength of the PBAT composite increased, albeit from a very low base level. This result was unexpected as the reinforcing potential of natural fibres in biopolyester has previously been demonstrated, e.g., for TMP fibre in PLA [13] and flax fibre in PLA [29].

The addition of MDF fibres did not improve the impact strength (Figure 2) but caused a moderate (PLA) to significant decrease (PBS, PBSA) of impact strength compared to the plain biopolyesters. Plain PBAT did not break under the testing conditions employed.

When MDF fibres were incorporated within a PP matrix with the addition of a compatibiliser such as MA-PP, a substantial tensile strength increase and impact strength decrease are observed $[2,30]$. Therefore, the decrease of both values with the MDF filled biopolyesters was unexpected and prompted us to investigate why MDF fibres were unable to reinforce the biopolyesters.

3.2. Fibre Length. One hypothesis for the lack of reinforcement was a drastic reduction of the MDF fibre aspect ratio. 


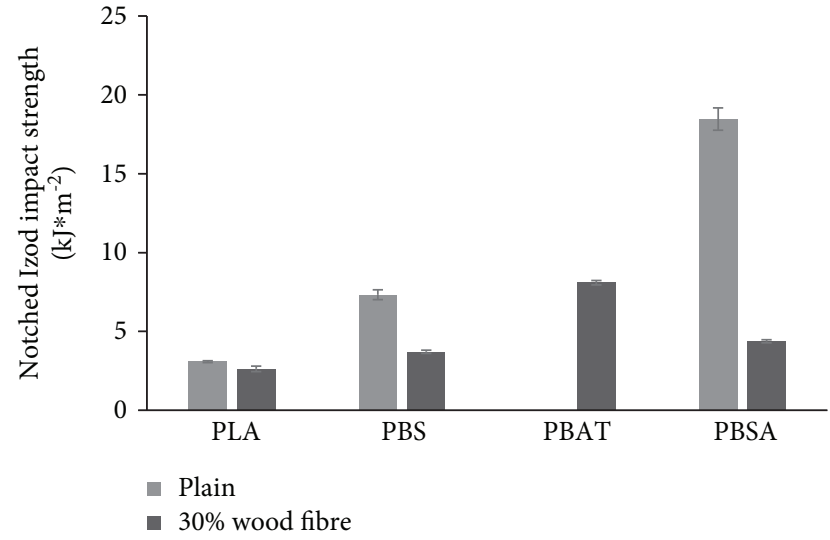

FIgURE 2: Impact strength of 4 biopolyesters, plain and filled with 30 wt $\%$ MDF fibre (error bars $= \pm 95 \%$ confidence interval) $(n=10)$.

Extrusion compounding and consequent injection moulding typically lead to shortening of natural fibres [13, 31]. Typically, when processed with PP, loose MDF fibre undergoes a fibre length reduction from $1.7-1.5$ down to $0.5-0.3 \mathrm{~mm}$ $[31,32]$. Therefore, the possibility of further MDF fibre length reduction during their processing with biopolyester was tested. It was found that after injection moulding with all biopolyesters, the MDF fibre length was reduced to $0.4 \mathrm{~mm}$ (data not shown). The greater decrease in fibre length with PLA compared to PP has been reported by Peltola et al. [13] and attributed to the higher viscosity of the PLA. However, even at a fibre length of $0.4 \mathrm{~mm}$ and an aspect ratio of 8.8 , some reinforcement potential would be expected from the fibres. For example, Guo et al. [33] added a range of wood fibres of a similar length to PP and showed increases in strength, modulus, and impact. Therefore, the fibre attrition was considered insufficient to explain the reduction of tensile strength generated by the addition of MDF fibre in the biopolyester matrices.

3.3. Rheology. During extrusion and injection moulding, the biopolyesters were difficult to process in the presence of MDF fibres. This was most striking for PLA as it was not possible to pull strands from the extruder. These observations are consistent with a reduction of the polymer viscosities. Therefore, the melt viscosity behaviours of PLA and PLA containing MDF fibre were compared using a rheometer mimicking extrusion conditions $\left(180^{\circ} \mathrm{C}, 1 \mathrm{~Hz}\right)$. The addition of MDF fibre reduced the melt viscosity of the plain PLA by half (Figure 3). This observation was unexpected as the addition of MDF fibres typically increases PP melt viscosity [13]. PLA, containing MDF fibre, is seven times less viscous than its PP reference counterpart. The low melt viscosity of PLA/MDF fibres explains its unstable behaviour during extrusion (Figure 3).

3.4. Molecular Weight. Polymers typically show a correlation between viscosity and molecular weight (MW) as described by a power law [34]: $\eta_{0} \propto M^{3.4}$, where $\eta_{0}$ is the melt viscosity and $M$ is the molecular weight.

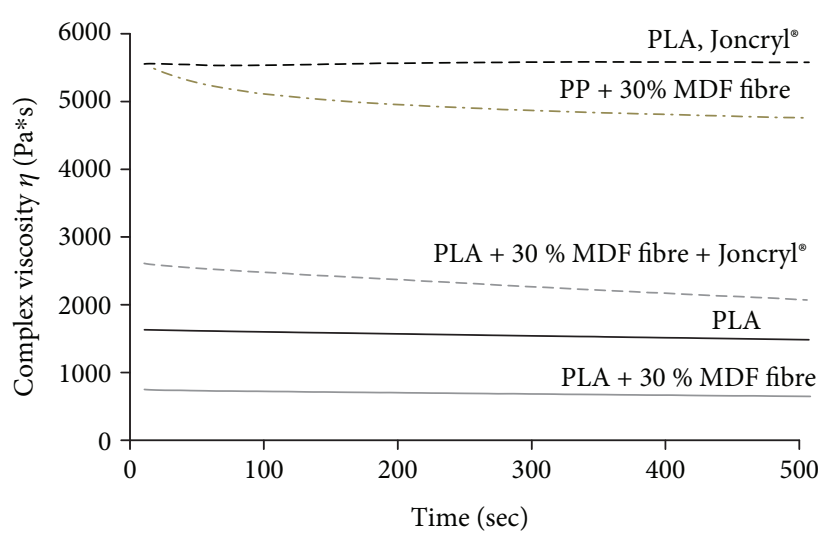

Figure 3: Apparent viscosity of plain PLA and PLA filled with $30 \mathrm{wt} \%$ MDF fibre $\left(180^{\circ} \mathrm{C}\right.$ and $\left.1 \mathrm{~Hz}\right)$. The same materials containing Joncryl ${ }^{\circledR}$, a chain extender, are also represented. For reference, PP containing $30 \mathrm{wt} \% \mathrm{MDF}$ fibre is included.

Rheology observations were compared to molecular weight by assessing molecular weight distributions with gel permeation chromatography (GPC). The extrusion and injection moulding steps did not affect plain PLA and PBSA but led to a 20 and $28 \%$ MW reduction for PBS and PBAT, respectively (Figure 4). The presence of MDF fibre during processing negatively affected the MW of all biopolymers with the most drastic effect observed for PLA. After injection moulding, there was a $36 \%$ reduction in MW of PLA filled with MDF fibres compared to plain PLA. Overall, it was found that, in accordance with the power law, the magnitude of molecular weight reduction of PLA containing MDF fibres (Figure 4) was consistent with its reduction in viscosity (Figure 3).

Next, it was investigated whether the molecular weight reduction of biopolyesters due to MDF fibres could be observed with other lignocellulosic fibres. PLA was selected as it exhibits the strongest MW decrease during compounding in the presence of MDF fibres. Bleached kraft fibres and flax fibres were the lignocellulosic fibres used for comparison. Bleached Pinus radiata kraft fibres were selected as they had the same origin as the MDF fibres but differed by their high cellulose content, having only traces of lignin and hemicelluloses [35]. The flax fibres, a nonwood fibre with very low lignin content [36], represents an agricultural fibre that often directly competes against wood fibre. Both kraft and flax fibres caused a slight MW reduction of PLA during extrusion and injection moulding, but neither of the fibres had an effect as large as that of MDF fibres on PLA (Figure 5).

3.5. Influence of Chain Extender Additives on PLA's Molecular Weight during Compounding. Substantial degradation of the PLA polymer was specifically observed when processed with MDF fibres. Compensation for this detrimental effect on MW and mechanical properties was explored by using chain extender additives to counteract this degradation during extrusion compounding. Two commercial chain extenders were chosen: BioAdimide ${ }^{\circledR}$ which is a product specifically designed to improve melt stability of biobased polyesters and Joncryl ${ }^{\circledR}$, a BASF product designed to reverse the 


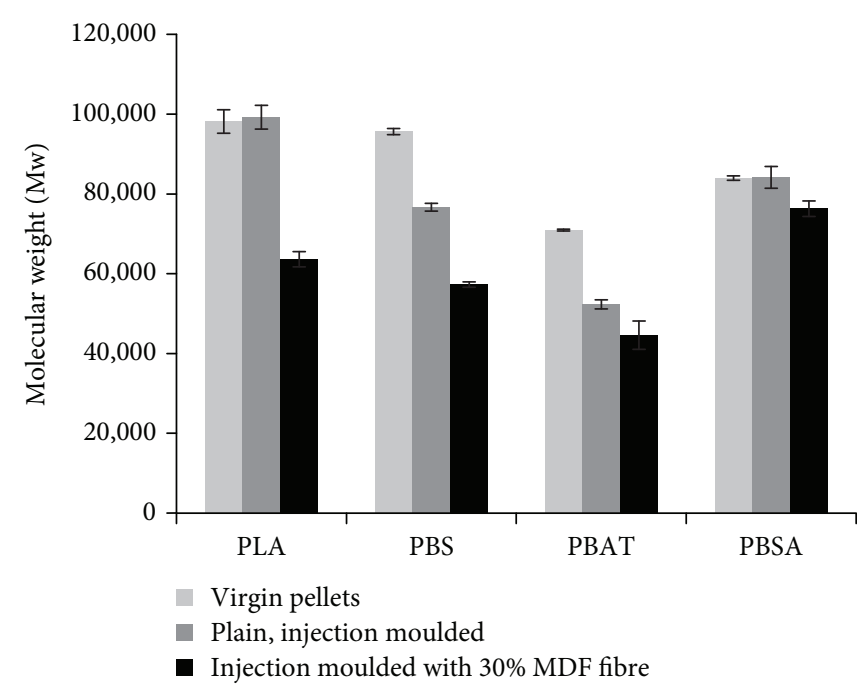

Figure 4: Molecular weight reduction of four biopolyesters before and after extrusion with or without $30 \mathrm{wt} \%$ MDF fibres (error bars $= \pm 95 \%$ confidence interval $)(n=2)$.

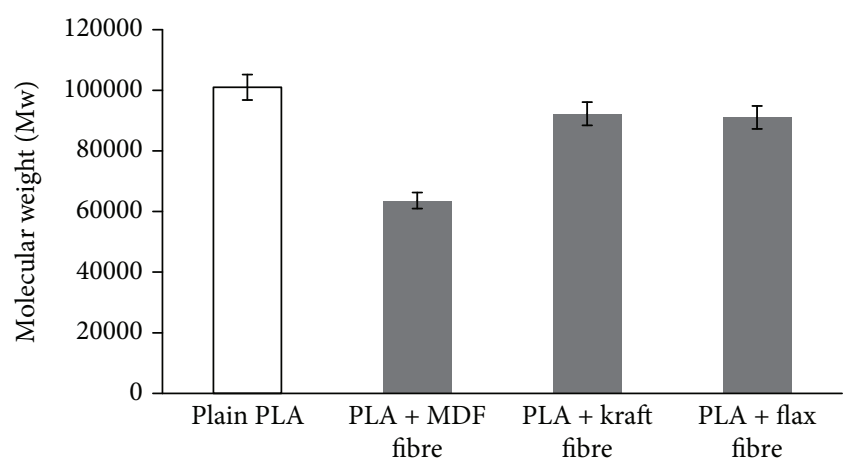

Figure 5: Effect of three natural fibre types on PLA MW after extrusion and injection moulding (all fibres at $30 \mathrm{wt} \%$ ) (error bars $= \pm 95 \%$ confidence interval).

degradation of poly-ethylene-terephthalate (PET) and polyester packaging products.

The addition of both chain extenders during extrusion led to an increase of molecular weight beyond the original MW of PLA (Figure 6). The strongest effect was observed with the addition of Joncryl ${ }^{\circledR}$ leading to a $150 \%$ increase compared with the plain PLA MW. In the presence of MDF fibres, the addition of chain extenders leads to a doubling of the MW value, with values greater than that of plain PLA. This was reflected by the improved processability of the materials. Melt viscosity was high enough (Figure 3), to allow strands to be pulled from the extruder.

It is unclear why the MDF fibres led the molecular weight decrease of PLA during compounding. When comparing MDF to bleached kraft pulp and flax fibres, the main difference is the presence of lignin. Softwood bleach kraft pulp and MDF fibres contain 0.8 and $31.2 \%$ of lignin, respectively [9]. Several studies have shown a positive effect of lignin in PLA blends. Lignin greatly improved the thermal stability

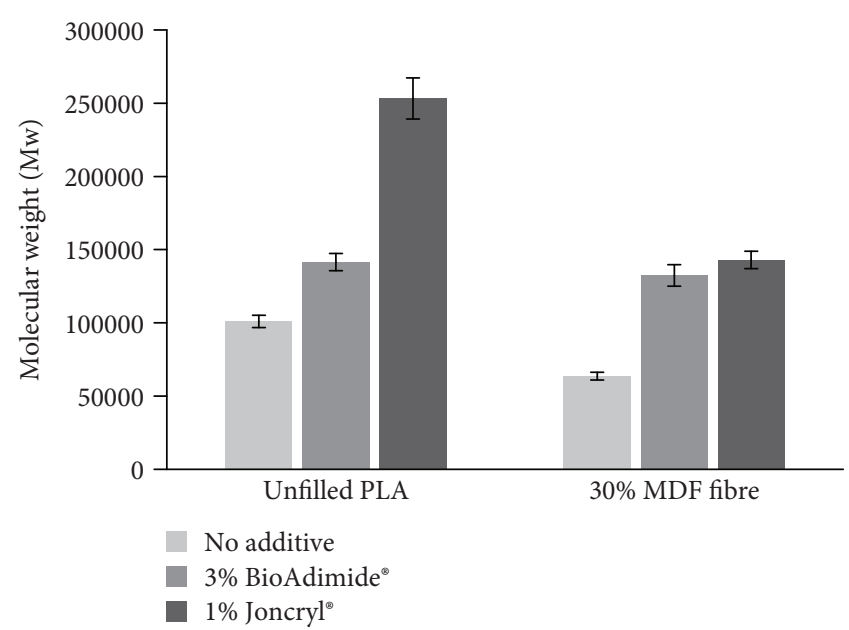

FIGURE 6: Influence of chain extenders on PLA molecular weight (error bars $= \pm 95 \%$ confidence interval) $(n=2)$.

of PLA, and acetylated lignins appear to prevent hydrolytic degradation of PLA [37]. Another difference is the relatively high acidity of MDF fibre ( $\mathrm{pH} 4.0)$ [38] compared to kraft ( $\mathrm{pH}$ 5.5) and flax (pH 5.5). However, lactic acid $\mathrm{pKa}$ is 3.84 [39] and it was reported that PLA was the most stable at $\mathrm{pH}=4$ [40]. Therefore the $\mathrm{pH}$ of the MDF fibre cannot be solely responsible for molecular weight reduction. Finally, extractives are virtually absent from bleached kraft pulp [9], while due to the high temperature used during the softwood MDF manufacture process, the MDF fibre contains high levels of water and DCM extractives, i.e., 7.2 and $0.4 \mathrm{wt} \%$, respectively [41]. A GC-MS study showed that MDF fibre extractives contain a wide range of triglycerides, sterol esters, fatty acids, and resin acids [42]. It can be hypothesised that these extractives were released during extrusion and led to the degradation of the PLA chains. The unique high levels of extractives in MDF would also explain why it has been possible to reinforce PLA with TMP without using additives [13]. TMP is refined at lower temperatures which leads to a substantial reduction in extractives [41].

3.6. Influences of Chain Extender Additives on PLA NFC's Mechanical Properties. As the ability of chain extenders to increase the molecular weight of PLA containing MDF fibres was demonstrated, the next step was to determine if this effect was translated into an improvement of NFC's mechanical properties. Wood flour, a low aspect ratio filler, was used as a comparison to MDF fibre. Young's modulus of the plain PLA was increased by the presence of MDF fibre (Figure 1) and wood flour (Figure 7), but neither of the additives was able to significantly increase this effect further (Figure 7).

Despite leading to the increased molecular weight of PLA, the addition of BioAdimide ${ }^{\circledR}$ did not translate into an improvement of mechanical strength in either NFC (Figure 7). It has previously been found [43] that the molecular weight of amorphous PLA does not always correlate with its tensile strength. The addition of $30 \mathrm{wt} \% \mathrm{MDF}$ and $1 \mathrm{wt} \%$ Joncry ${ }^{\circledR}$ was the only combination able to increase the tensile strength of PLA $(+16 \%)$. This effect was not seen with the 

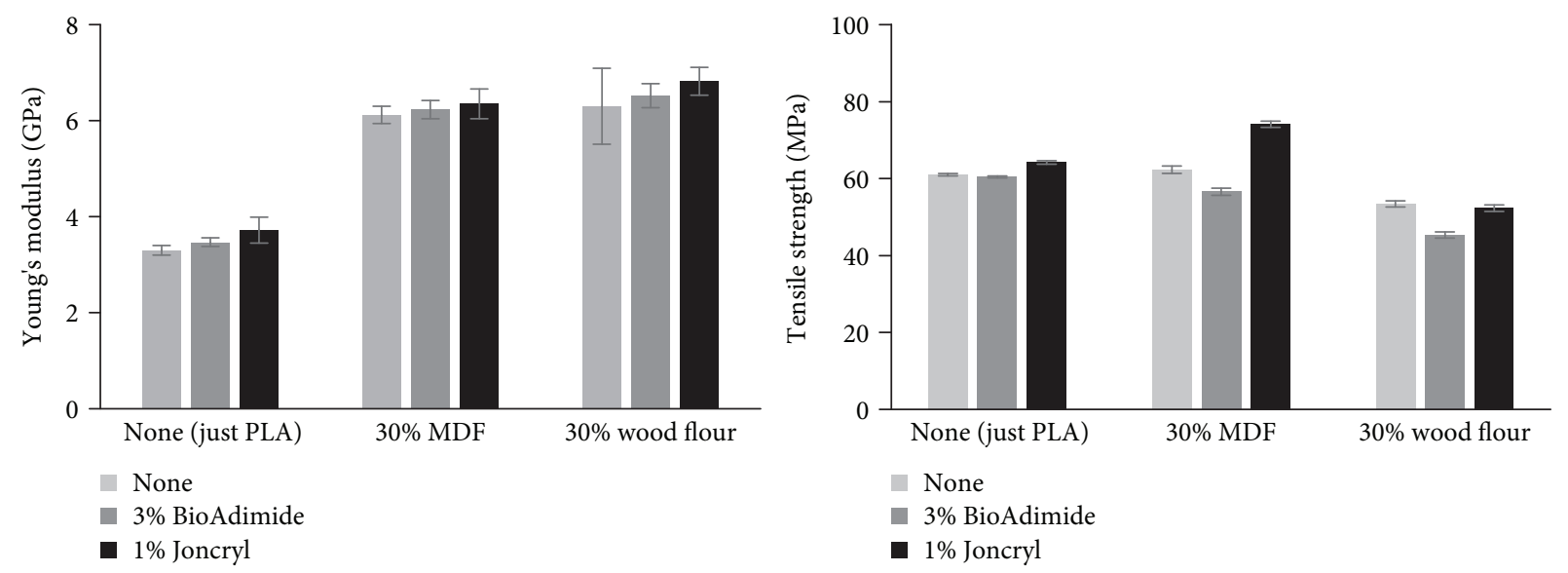

FIGURE 7: Effect of chain extender additives on tensile modulus and maximum tensile strength of injection moulded PLA NFC test samples. $1 \mathrm{wt} \%$ Joncryl $^{\circledR}$ and $3 \mathrm{wt} \%$ BioAdimide ${ }^{\circledR}$, on the modulus and strength of a plain PLA. PLA containing $30 \mathrm{wt} \%$ of MDF fibre and PLA containing $30 \mathrm{wt} \%$ wood flour (error bars $= \pm 95 \%$ confidence interval) $(n=10)$.

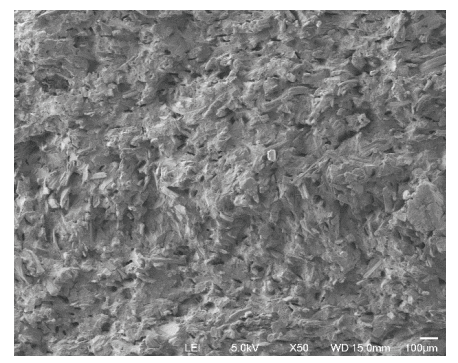

(a)

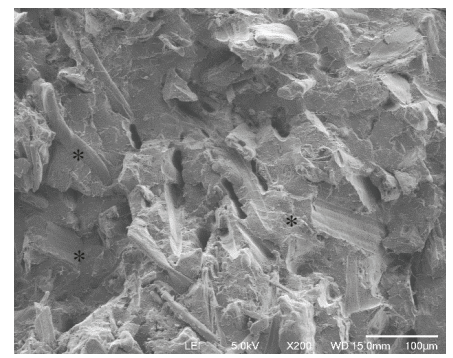

(d)

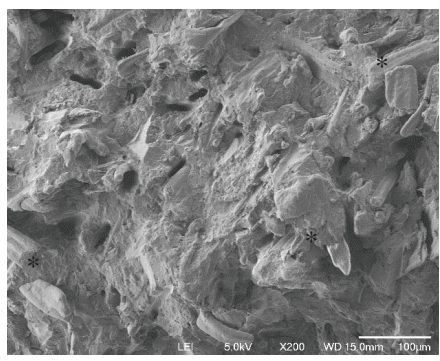

(b)

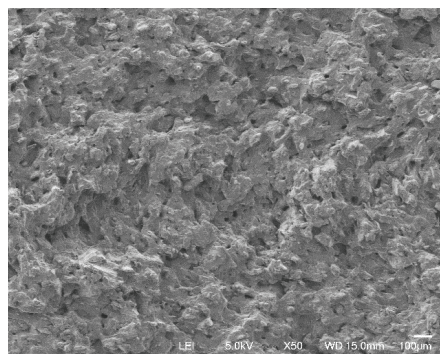

(e)

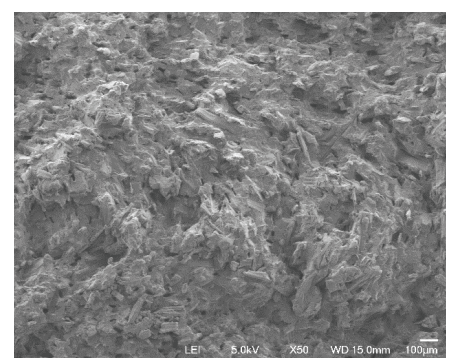

(c)

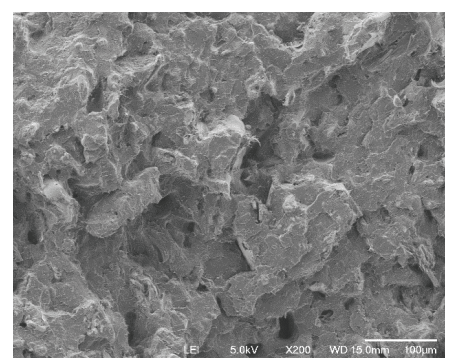

(f)

FiguRE 8: Scanning electron microscopy images of fracture surfaces; effect of chain extender additives on fibre pull-out. (a, b) Plain PLA containing $30 \mathrm{wt} \%$ of MDF fibre. (c, d) PLA containing $30 \mathrm{wt} \%$ of MDF fibre and 3 wt \% BioAdimide ${ }^{\circledR}$. (e, f) PLA containing 30 wt $\%$ of MDF fibre and $1 \mathrm{wt} \%$ Joncryl $^{\circledR}$. Asterisks indicate a fibre pull-out. Scale bar $=100 \mu \mathrm{m}(n=1)$.

wood flour, as in all the cases, addition of wood flour leads to a decrease in tensile strength. This illustrates that tensile strength increase requires not a simple filler but high aspect ratio fibres.

3.7. Fractography Studies. The fracture surface of injection moulded specimens was observed by scanning electron microscopy (SEM) in order to understand why despite both chain extenders increasing the MW of the MDF fibre PLA composite, only Joncryl ${ }^{\circledR}$ is able to improve tensile properties (Figure 8). A large number of pulled-out fibres can be observed for samples without any additive and in the presence of $3 \mathrm{wt} \%$ BioAdimide $^{\circledR}$ (Figures $8(\mathrm{a})-8(\mathrm{~d})$ ). On the other hand, the addition of Joncryl ${ }^{\circledR}$ led to a near absence of visible fibre pull-out (Figures 8(e) and 8(f)). This observation was unexpected as Jaszkiewicz et al. [44] observed that the addition of $3 \mathrm{wt} \%$ Joncryl $^{\circledR} 3229$, with man-made cellulose (20 wt \%), manufactured by Cordenka, in a PLA matrice led to a massive fibre pull-out. The authors assumed that the maleinated groups of Joncryl ${ }^{\circledR} 3229$ reacted chemically with the hydroxyl groups of the cellulosic fibres making the fibres smoother and more slippery. In the case of this study, it can be assumed that Joncryl ${ }^{\circledR}$ is improving the interfacial adhesion between the fibre and the matrix which enabled the fibre to reinforce the matrix and led to the increase in tensile strength. 


\section{Conclusions}

In order to obtain a fully biodegradable composite, natural fibres need to be used as reinforcements of the biodegradable plastic matrix. Successful integration of cost competitive MDF fibre with biopolyesters is an attractive prospect.

(i) This study demonstrates that the addition of $30 \mathrm{wt}$ $\%$ of MDF fibre with a biopolyester matrix led to unexpected difficulties of compounding due to a reduction in melt viscosity

(ii) This phenomenon can be explained by the fact that MDF fibre reduced the MW of the four biopolyesters tested, with the strongest reduction observed for PLA

(iii) The MW reduction specifically caused by the addition of MDF fibre explains the absence of strength reinforcement of the biopolyester matrix

(iv) The addition of Joncryl ${ }^{\circledR}$, a chain extender, resulted in a PLA polymer with a higher MW than the starting PLA material

(v) Joncryl ${ }^{\circledR}$ reinforced the interface between PLA and MDF fibres

(vi) This PLA/MDF composite demonstrated an increase in strength

(vii) The MW reduction could be due to the presence of extractives in MDF fibre released during compounding

(viii) This work demonstrates the possibility to obtain biopolyester composites reinforced with MDF fibres

\section{Data Availability}

The underlying raw data for the charts presented can be obtained by request from the corresponding author.

\section{Conflicts of Interest}

Scion is the proprietor of a family of registered and licensed patents relating to a reinforcing wood fibre product and process for use in NFCs.

\section{Acknowledgments}

The authors would like to acknowledge Dawn Smith, Marc Gaugler, and Gareth Lloyd-Jones for their extensive review of this manuscript. This work was funded by the New Zealand Ministry of Business, Innovation and Employment (MBIE).

\section{References}

[1] K. L. Pickering, M. G. A. Efendy, and T. M. Le, "A review of recent developments in natural fibre composites and their mechanical performance," Composites Part A: Applied Science and Manufacturing, vol. 83, pp. 98-112, 2016.
[2] A. Fernyhough and M. Markotsis, "Long biofibers and engineered pulps for high performance bioplastics and biocomposites," in Handbook of Bioplastics and Biocomposites Engineering Applications: Pilla/Handbook, pp. 555-579, John Wiley \& Sons, Inc., 2011.

[3] P. Nygård, B. S. Tanem, T. Karlsen, P. Brachet, and B. Leinsvang, "Extrusion-based wood fibre-PP composites: wood powder and pelletized wood fibres - a comparative study," Composites Science and Technology, vol. 68, no. 1516, pp. 3418-3424, 2008.

[4] C. M. Chan, L. J. Vandi, S. Pratt et al., "Composites of wood and biodegradable thermoplastics: a review," Polymer Reviews, vol. 58, no. 3, pp. 444-494, 2017.

[5] M. Sorieul, A. Dickson, S. Hill, and H. Pearson, "Plant fibre: molecular structure and biomechanical properties, of a complex living material, influencing its deconstruction towards a biobased composite," Materials, vol. 9, no. 8, p. 618, 2016.

[6] L. A. Donaldson and T. D. Lomax, "Adhesive/fibre interaction in medium density fibreboard," Wood Science and Technology, vol. 23, no. 4, pp. 371-380, 1989.

[7] Z. Shao and K. Li, "The effect of fiber surface lignin on interfiber bonding," Journal of Wood Chemistry and Technology, vol. 26, no. 3, pp. 231-244, 2007.

[8] A. McDonald, A. Clare, and B. Dawson, "Surface characterisation of Radiata pine high-temperature TMP fibres by X-ray photo-electron spectroscopy," in Proceeding of the 53rd General APPITA Conference, Rotorua, New Zealand, 1999.

[9] K. L. Spence, R. A. Venditti, Y. Habibi, O. J. Rojas, and J. J. Pawlak, "The effect of chemical composition on microfibrillar cellulose films from wood pulps: mechanical processing and physical properties," Bioresource Technology, vol. 101, no. 15, pp. 5961-5968, 2010.

[10] R. D. Burdon, R. P. Kibblewhite, and M. J. C. Riddell, "Wood density and kraft fibre and pulp properties of four Pinus radiata provenances," New Zealand Journal of Forestry Science, vol. 29, no. 2, pp. 214-224, 1999.

[11] A. R. Dickson and D. Sandquist, "Mode of wood fibre breakage during thermoplastic melt processing," Composites Part A: Applied Science and Manufacturing, vol. 112, pp. 496-503, 2018.

[12] O. Faruk, A. K. Bledzki, H. P. Fink, and M. Sain, "Biocomposites reinforced with natural fibers: 2000-2010," Progress in Polymer Science, vol. 37, no. 11, pp. 1552-1596, 2012.

[13] H. Peltola, E. Pääkkönen, P. Jetsu, and S. Heinemann, "Wood based PLA and PP composites: effect of fibre type and matrix polymer on fibre morphology, dispersion and composite properties," Composites Part A: Applied Science and Manufacturing, vol. 61, pp. 13-22, 2014.

[14] K. G. Satyanarayana, G. G. C. Arizaga, and F. Wypych, "Biodegradable composites based on lignocellulosic fibersan overview," Progress in Polymer Science, vol. 34, no. 9, pp. 982-1021, 2009.

[15] T. Gurunathan, S. Mohanty, and S. K. Nayak, "A review of the recent developments in biocomposites based on natural fibres and their application perspectives," Composites Part A: Applied Science and Manufacturing, vol. 77, pp. 1-25, 2015.

[16] T. L. do Amaral Montanheiro, F. R. Passador, M. P. de Oliveira, N. Durán, and A. P. Lemes, "Preparation and characterization of maleic anhydride grafted poly (hydroxybutirate-CO-hydroxyvalerate)-PHBV-g-MA," Materials Research, vol. 19, no. 1, pp. 229-235, 2016. 
[17] D. Plackett, "Maleated polylactide as an interfacial compatibilizer in biocomposites," Journal of Polymers and the Environment, vol. 12, no. 3, pp. 131-138, 2004.

[18] L. Quiles-Carrillo, N. Montanes, D. Garcia-Garcia, A. Carbonell-Verdu, R. Balart, and S. Torres-Giner, "Effect of different compatibilizers on injection-molded green composite pieces based on polylactide filled with almond shell flour," Composites Part B: Engineering, vol. 147, pp. 76-85, 2018.

[19] M. Kazayawoko, J. J. Balatinecz, R. T. Woodhams, and S. Law, "Effects of wood fiber surface chemistry on the mechanical properties of wood fiber-polypropylene composites," International Journal of Polymeric Materials and Polymeric Biomaterials, vol. 37, no. 3-4, pp. 237-261, 2006.

[20] A. Jaszkiewicz, A. Meljon, A. K. Bledzki, and M. Radwanski, "Gaining knowledge on the processability of PLA-based short-fibre compounds - a comprehensive comparison with their PP counterparts," Composites Part A: Applied Science and Manufacturing, vol. 83, pp. 140-151, 2016.

[21] L. Zhang, S. Lv, C. Sun, L. Wan, H. Tan, and Y. Zhang, "Effect of MAH-g-PLA on the properties of wood fiber/polylactic acid composites," Polymer, vol. 9, no. 11, 2017.

[22] K. M. Bogren, E. K. Gamstedt, R. C. Neagu, M. AÅkerholm, and M. LindstroÖm, "Dynamic-mechanical properties of wood-fiber reinforced polylactide: experimental characterization and micromechanical modeling," Journal of Thermoplastic Composite Materials, vol. 19, no. 6, pp. 613-637, 2006.

[23] B. L. Shah, S. E. Selke, M. B. Walters, and P. A. Heiden, "Effects of wood flour and chitosan on mechanical, chemical, and thermal properties of polylactide," Polymer Composites, vol. 29, no. 6, pp. 655-663, 2008.

[24] L. Wei and A. McDonald, "A review on grafting of biofibers for biocomposites," Materials, vol. 9, no. 4, p. 303, 2016.

[25] B. A. Ahmed Ali, S. M. Sapuan, E. S. Zainudin, and M. Othman, "Implementation of the expert decision system for environmental assessment in composite materials selection for automotive components," Journal of Cleaner Production, vol. 107, pp. 557-567, 2015.

[26] A. El-Sabbagh, "Effect of coupling agent on natural fibre in natural fibre/polypropylene composites on mechanical and thermal behaviour," Composites Part B: Engineering, vol. 57, pp. 126-135, 2014.

[27] ASTM International, "Standard test method for tensile properties of plastics," ASTM International, Pennsylvania, USA, 2010.

[28] ASTM International, "Standard test methods for determining the Izod pendulum impact resistance of plastics," ASTM International, Pennsylvania, USA, 2010.

[29] K. Oksman, M. Skrifvars, and J. F. Selin, "Natural fibres as reinforcement in polylactic acid (PLA) composites," Composites Science and Technology, vol. 63, no. 9, pp. 1317-1324, 2003.

[30] M. D. H. Beg and K. L. Pickering, "Mechanical performance of Kraft fibre reinforced polypropylene composites: influence of fibre length, fibre beating and hygrothermal ageing," Composites Part A Applied Science and Manufacturing, vol. 39, no. 11, pp. 1748-1755, 2008.

[31] M. D. H. Beg and K. L. Pickering, "Reprocessing of wood fibre reinforced polypropylene composites. Part I: effects on physical and mechanical properties," Composites Part A Applied Science and Manufacturing, vol. 39, no. 7, pp. 10911100, 2008.
[32] A. R. Dickson, D. Even, J. M. Warnes, and A. Fernyhough, "The effect of reprocessing on the mechanical properties of polypropylene reinforced with wood pulp, flax or glass fibre," Composites Part A: Applied Science and Manufacturing, vol. 61, pp. 258-267, 2014.

[33] Q. Guo, B. Cheng, M. Kortschot et al., "Performance of long Canadian natural fibers as reinforcements in polymers," Journal of Reinforced Plastics and Composites, vol. 29, no. 21, pp. 3197-3207, 2010.

[34] P. J. Flory and T. G. Fox, "Treatment of intrinsic viscosities," Journal of the American Chemical Society, vol. 73, no. 5, pp. 1904-1908, 1951.

[35] J. Laine, P. Stenius, G. Carlsson, and G. Ström, "The effect of ECF and TCF bleaching on the surface chemical composition of kraft pulp as determined by ESCA," Nordic Pulp and Paper Research Journal, vol. 11, no. 3, pp. 201-210, 1996.

[36] A. Lefeuvre, A. Bourmaud, C. Morvan, and C. Baley, "Elementary flax fibre tensile properties: correlation between stressstrain behaviour and fibre composition," Industrial Crops and Products, vol. 52, pp. 762-769, 2014.

[37] O. Gordobil, I. Egüés, R. Llano-Ponte, and J. Labidi, "Physicochemical properties of PLA lignin blends," Polymer Degradation and Stability, vol. 108, pp. 330-338, 2014.

[38] C. Xing, S. Y. Zhang, J. Deng, B. Riedl, and A. Cloutier, "Medium-density fiberboard performance as affected by wood fiber acidity, bulk density, and size distribution," Wood Science and Technology, vol. 40, no. 8, pp. 637-646, 2006.

[39] A. M. Eyal and R. Canari, "pH dependence of carboxylic and mineral acid extraction by amine-based extractants: effects of pKa, amine basicity, and diluent properties," Industrial and Engineering Chemistry Research, vol. 34, no. 5, pp. 17891798, 1995.

[40] M. A. Elsawy, K. H. Kim, J. W. Park, and A. Deep, "Hydrolytic degradation of polylactic acid (PLA) and Its composites," Renewable and Sustainable Energy Reviews, vol. 79, pp. 13461352, 2017.

[41] K. D. Murton, "The effect of preheating and refining conditions on radiata pine MDF fibre quality," in 52nd Appita annual conference, Melbourne, 1998.

[42] A. G. McDonald, T. R. Stuthridge, A. B. Clare, and M. J. Robinson, "Isolation and analysis of extractives from radiata pine HTMP fibre," in 9th International Symposium of Wood and Pulping Chemistry, pp. 9-12, Montreal, Canada, June 1997.

[43] G. Perego, G. D. Cella, and C. Bastioli, "Effect of molecular weight and crystallinity on poly(lactic acid) mechanical properties," Journal of Applied Polymer Science, vol. 59, no. 1, pp. 37-43, 1996.

[44] A. Jaszkiewicz, A. K. Bledzki, and P. Franciszczak, "Improving the mechanical performance of PLA composites with natural, man-made cellulose and glass fibers- a comparison to PP counterparts," Polimery, vol. 58, no. 6, pp. 435-442, 2013. 


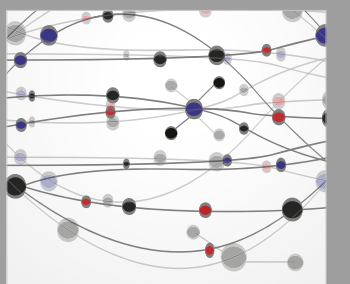

The Scientific World Journal
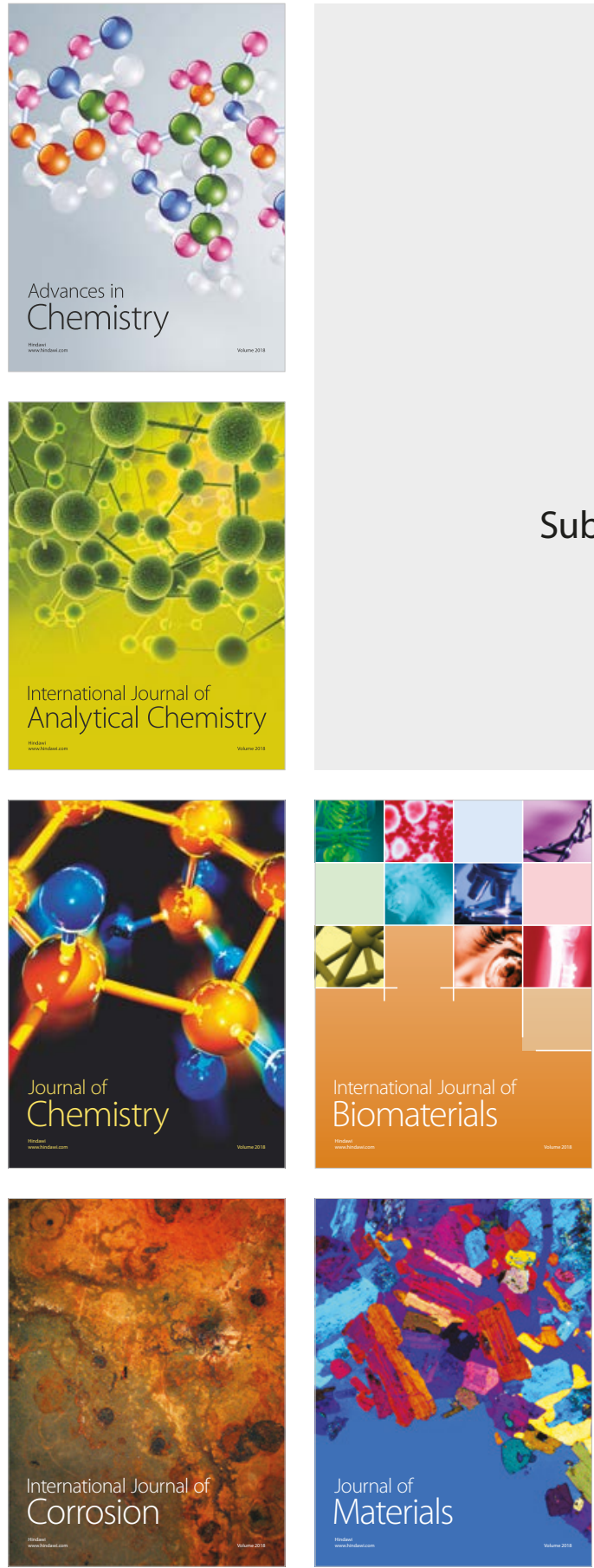

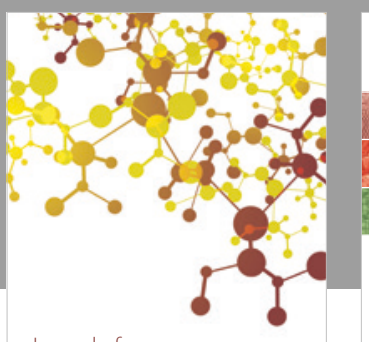

Journal of

Applied Chemistry
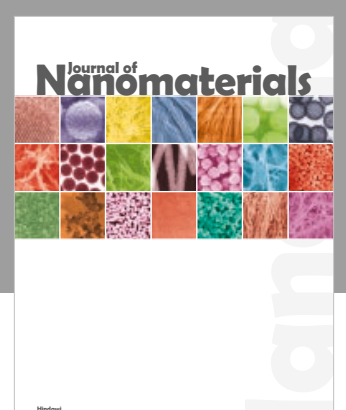

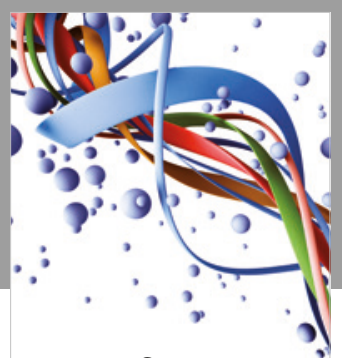

Scientifica

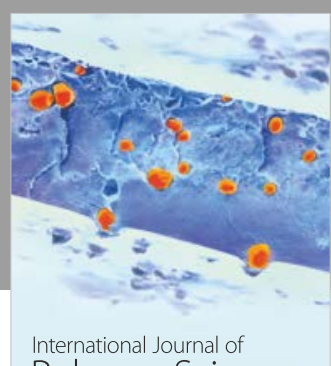

Polymer Science

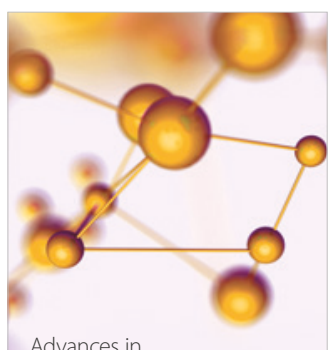

Physical Chemistry
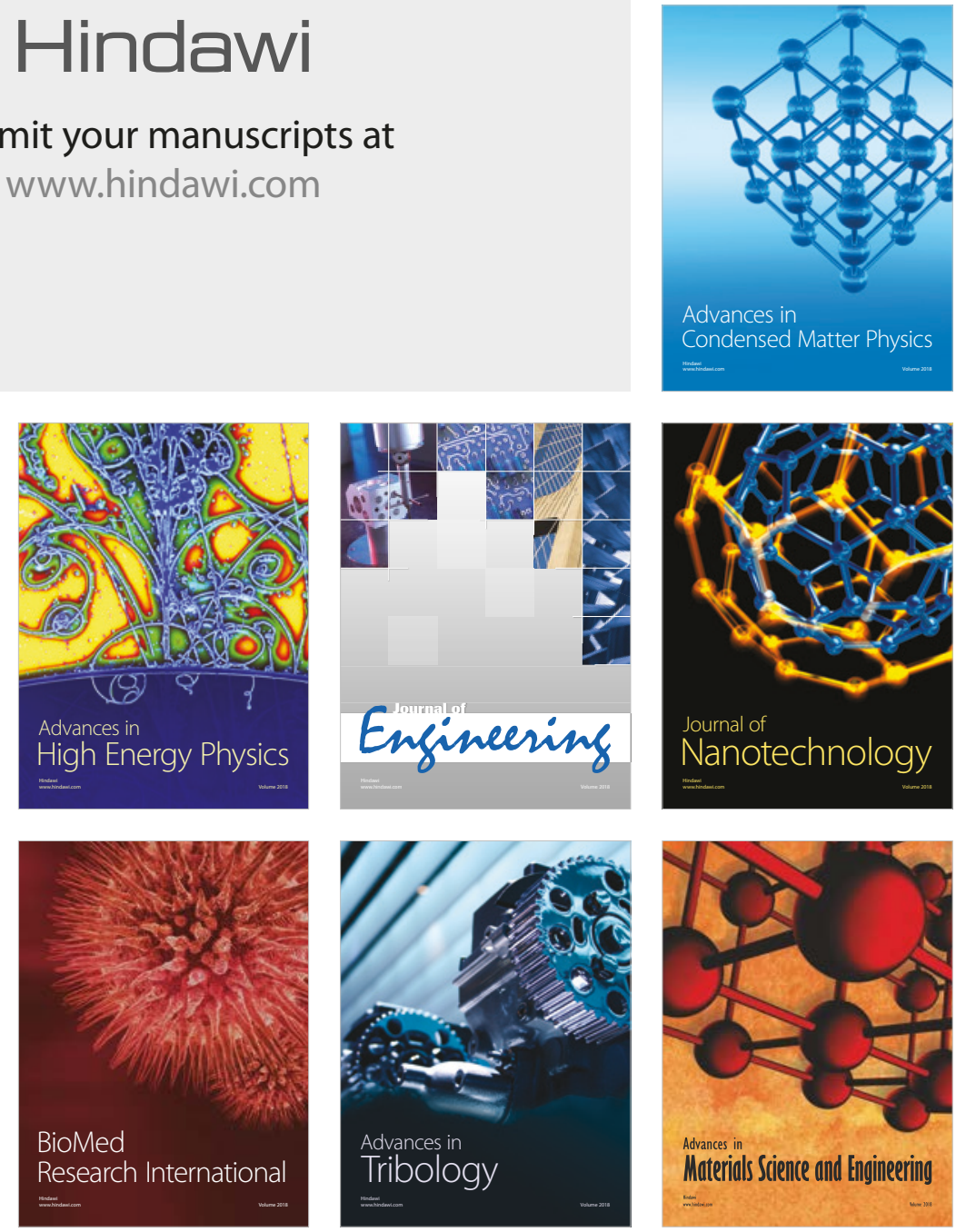